\title{
Solubilization and penetration enhancement of water- soluble and amphiphilic phospholipid polymer aggregate for poorly soluble drugs
}

\author{
Tomohiro Konno ${ }^{1 *}$ and Kazuhiko Ishihara ${ }^{1,2 *}$ \\ ${ }^{1}$ Department of Bioengineering, School of Engineering, The University of Tokyo, Japan \\ ${ }^{2}$ Department of Materials Engineering, School of Engineering, The University of Tokyo, Japan
}

It is important to develop the solubilizing reagents for the poorly soluble drugs to achieve safer pharmacological therapy including solid cancer treatment. Indeed, these drug candidates have low solubility in an aqueous medium [1]. Also, it has been suggested that to achieve an effective anticancer treatment, the drugs must penetrate into the tissue efficiently [2]. Therefore, it is required to prepare the solubilizing reagents with good tissue penetration enhancement for these drugs. Water-soluble polymeric nanomaterials are one of the candidates as the solubilizing reagent due to their controllable molecular design from aspects of both solubilization ability for the drugs and cytocompatibility in the living system. In this mini-review, we summarize recent developments of water-soluble and amphiphilic phospholipid polymers, which possess the same polar group of a natural phospholipid and hydrophobic moiety to enhance solubilization of the poorly soluble drugs in an aqueous medium.

Currently, the phospholipid polymers, 2-methacryloyloxyethyl phosphorylcholine (MPC) polymers, are widely applied to the implantable biomedical devices due to their excellent biocompatibility for long-term. We have been developed several MPC polymers by copolymerization of MPC with hydrophobic monomer such as $n$-butyl methacrylate (BMA) by a conventional radical polymerization technique. The water solubility of the poly(MPC-co-BMA (PMB))s can be controlled by their molecular weight and composition of monomer units in the copolymer. The PMB contained below $30 \mathrm{~mol} \%$ of MPC units (PMB30), the polymer is water-insoluble, and they can be used as a surface modification material on the solid substrates by a simple solution coating technique. This PMB30 has been approved by Food and Drug Administration (FDA), USA and applied for implantable medical devices worldwide.

On the other hand, even in the case of $30 \mathrm{~mol} \%$ of MPC units, the molecular weight is lower than $50 \mathrm{kDa}$, the polymer can be dissolved in water due to super-hydrophilic nature of the MPC unit [3]. Especially, the water-soluble and amphiphilic PMB30, named PMB30W, can form a stable polymer aggregate with $20-30 \mathrm{~nm}$ in diameter in an aqueous medium through the hydrophobic interaction between polymer chains, and act as a solubilizing reagent for poorly soluble drugs [35]. Noteworthy, the PMB30W aggregates are very stable even under the biological condition, and the polarity in the aggregate is as same as that of ethanol, which is good solvent for many poorly soluble drugs. Paclitaxel (PTX) is approved for ovarian, breast and lung, bladder, prostate, melanoma, esophageal, and other types of solid tumor cancers as well as Kaposi's sarcoma. As the solubility of PTX in water is very low (less than $0.3 \mu \mathrm{g} / \mathrm{mL}$ ), general commercial injection solutions of PTX needed a specific pharmaceutical emulsifier, such as Cremophor EL ${ }^{\circ}$ (CrEL). However, serious hypersensitive reactions have been reported due to the proportion of CrEL in the PTX formulation, which is significantly higher than that in other drug formulations. The PMB30W can dissolve the PTX in the concentration range over $1.0 \mathrm{mg} / \mathrm{mL}$ in an aqueous medium. Therefore, the water-soluble and amphiphilic PMB30W considered to be a good candidate for solubilizing reagent for the PTX due to good solubilization function and biocompatible property of the polymer [5].

The water-soluble and amphiphilic MPC polymers including the PMB30W have been reported that cell membrane penetration property, which is the non-toxic and mild penetration ability into the cell membrane [6,7]. In these cases, cytotoxicity and cell membrane damage does not express although the amphiphilic nature of the MPC polymers. The in vivo anti-cancer activity of PTX solubilized in PMB30W aggregates has been examined. Soma et al. reported that the PTX solubilized in the PMB30W aggregates demonstrates that intraperitoneal administration of the PMB30W formulation can elicit much stronger antitumor activity on peritoneal metastasis than that of a conventional PTX formulation using with CrEL, presumably because of its characteristics to accumulate more preferentially in disseminate nodules [8]. Also, Kamei et al. reported that the PTX formulation using with $\mathrm{PMB} 30 \mathrm{~W}$ aggregates demonstrate high accumulation in disseminated nodules, especially in hypovascular areas in the peripheral part of peritoneal nodules, presumably due to its superior penetrating activity directly into malignant tissue [9]. Tamura et al. reported that intravesical administration of PTX solubilized in PMB30W aggregates treatment exhibits significant tumor suppression in an orthotopic bladder cancer model compared to conventional formulation and that efficient penetration of the PMB30W into tumor tissue seemed to be associated with its antitumor effect [10].

These antitumor effects are resulted not only pharmaceutical activity caused by PTX but also solubility enhancement effects by the

${ }^{*}$ Correspondence to: Tomohiro Konno and Kazuhiko Ishihara, School of Engineering, The University of Tokyo, 7-3-1, Hongo, Bunkyo-ku, Tokyo 1138656, Japan, E-mail: konno@bioeng.t.u-tokyo.ac.jp / ishihara@mpc.t.u-tokyo. ac.jp

Received: July 23, 2018; Accepted: August 16, 2018; Published: August 20, 2018 
amphiphilic and cytocompatible nature of the PMB30W. It has no doubt that the pharmaceutical activity is depended on the specific drug itself, however, the tissue penetration effect might be regulated by solubilizing reagent and cell/tissue penetration enhancer such as PMB30W aggregates.

\section{References}

1. Lipinski CA (2000) Drug-like properties and the causes of poor solubility and poor permeability. J Pharmacol Toxicol Methods 44: 235-249. [Crossref]

2. Minchinton AI, Tannock IF (2006) Drug penetration in solid tumours. Nat Rev Cancer 6: 583-592. [Crossref]

3. Ishihara K, Iwasaki Y, Nakabayashi N (1999) Polymeric lipid nanosphere consisting of water-soluble poly(2-methacryloyloxyethyl phosphorylcholine-co-n-butyl methacrylate). Polym J 31: 1231-1236.

4. Konno T, Watanabe J, Ishihara K (2003) Enhanced solubility of paclitaxel using watersoluble and biocompatible 2-methacryloyloxyethyl phosphorylcholine polymers. $J$ Biomed Mater Res A 65: 209-214. [Crossref]
5. Ishihara $\mathrm{K}, \mathrm{Mu} \mathrm{M}$, Konno $\mathrm{T}$ (2018) Water-soluble and amphiphilic phospholipid copolymers having 2-methacryloyloxyethyl phosphorylcholine units for the solubilization of bioactive compounds. J Biomater Sci Polym Ed 29: 844-862. [Crossref]

6. Goda T, Goto Y, Ishihara K (2010) Cell-penetrating macromolecules: Direct penetration of amphipathic phospholipid polymers across plasma membrane of living cells. Biomaterials 31: 2380-2387. [Crossref]

7. Kojima R, Kasuya MCZ, Ishihara K, Hatanaka K (2011) Physicochemical delivery of amphiphilic copolymers to specific organelles. Polym J 43: 718-722.

8. Soma D, Kitayama J, Konno T, Ishihara K, Yamada J, et al. (2009) Intraperitoneal administration of paclitaxel solubilized with poly(2-methacryloxyethyl phosphorylcholine-co-n-butyl methacrylate) for peritoneal dissemination of gastric cancer. Cancer Sci 100: 1979-1985. [Crossref]

9. Kamei N, Kitayama J, Yamaguchi H, Soma D, Emoto S, et al. (2010) Spatia distribution of intraperitoneally administrated paclitaxel nanoparticles solubilized with poly(2-methacryloyloxyethyl phosphorylcholine-co-n-butyl methacrylate) in peritoneal metastatic nodules. Cancer Sci 102: 200-205. [Crossref]

10. Tamura K, Kikuchi E, Konno T, Ishihara K, Matsumoto K, et al. (2015) Therapeutic effect of intravesical administration of paclitaxel solubilized with poly(2methacryloyloxyethyl phosphorylcholine-co-n-butyl methacrylate) in an orthotopic bladder cancer model. BMC Cancer 15: 317. [Crossref]

Copyright: (C2018 Konno T. This is an open-access article distributed under the terms of the Creative Commons Attribution License, which permits unrestricted use, distribution, and reproduction in any medium, provided the original author and source are credited. 